\title{
Role of the Lens Thickness and the Surface Termination in the Formation of Subwavelength Images by a Negative-Index Photonic-Crystal Slab
}

\author{
Rubén Ortuño, Carlos García-Meca and Alejandro Martínez
}

Valencia Nanophotonics Technology Center, Universidad Politécnica de Valencia, Spain

\begin{abstract}
A two-dimensional photonic-crystal engineered to possess a refractive index equal to minus one at a certain frequency is used as a flat superlens. Subwavelength imaging (image size below half a wavelength) is observed both in the near-field and far-field regimes when the interface with air is properly terminated to support surface states with even symmetry.
\end{abstract}

\section{INTRODUCTION}

Imaging of an electromagnetic (EM) point source can be carried out by use of a flat slab of an effective medium with a refractive index of -1 , as proposed years ago by Veselago [1]. By analyzing the EM properties of a substance in which the relative electric permittivity $\varepsilon$ and magnetic permeability $\mu$ were both equal to -1 , Veselago obtained that the refracted angle at the interface of the analyzed substance with vacuum was identical to the incident angle but at the same side of the normal. Such a phenomenon is usually known as negative refraction. In addition, this hypothetical negative index medium (NIM) would be impedance matched with vacuum, thus avoiding the undesired reflection of energy at the interfaces. More recently, Pendry suggested that such a slab with $\varepsilon=\mu=-1$ would allow the imaging of the point source with an ideally perfect resolution, since a slab made of this substance would restore not only the phase of the propagating waves (PWs) but also the amplitude of the evanescent waves (EWs) [2]. This would allow overcoming the diffraction limit (which imposes a limit in the achievable resolution when using conventional optics) and, as a result, obtaining a subwavelength image of the source. Although it has been demonstrated that a perfect image cannot be recovered at the other side of the NIM slab due to the inherent losses and dispersion of the material as well as the finite size of the slab [3], it is now widely accepted that subwavelength resolution below the diffraction limit can be obtained by use of a NIM slab.

Natural materials do not possess a negative index of refraction so a NIM has to be constructed from artificiallyarranged composites. One choice is to engineer a substance with a negative effective $\mu$ (e.g. by arranging a periodic lattice of split-ring resonators) [4] and mix it with another engineered substance with a negative effective $\varepsilon$ (e.g. a periodic lattice of metallic wires) [5]. Following this approach, the negative refraction phenomenon predicted by Veselago has been observed at microwave frequencies [6]. Another choice to build a medium that displays negative refraction

*Address correspondence to this author at the Nanophotonics Technology Center, Universidad Politécnica de Valencia, Spain; Tel: +34963879768; Fax: +34963877827; E-mail: amartinez@ntc.upv.es properties is by use of photonic crystals ( $\mathrm{PhCs}$ ), which have the added values of low losses (if metals and high loss dielectric materials are not involved) and straightforward scalability up to visible and infrared wavelengths [7]. Physics behind the negative index of refraction in PhCs are well different from that occurring in the hypothetical NIM studied by Veselago [1] and Pendry [2]. In a PhC the permittivity $\varepsilon$ is always locally positive whereas the permeability $\mu$ is equal to unit. EM propagation inside a $\mathrm{PhC}$ takes place in the form of Bloch waves governed by the photonic band diagram that relates the frequency and the wave vector for each EM mode. As a result of the periodicity of the $\mathrm{PhC}$, the photonic band diagram can become very intricate. Indeed, the $\mathrm{PhC}$ can provide the negative refraction for $\mathrm{PWs}$ required by the Veselago lens in certain spectral regions. Negative refraction (and its resulting imaging properties) in PhCs occurs under two conditions [8]: (i) at frequencies for which the equifrequency surfaces (EFSs) are placed in a corner of the first Brillouin zone, so that propagation is forbidden along one of the main symmetry direction (i.e., partial band gap) and allowed in the other one [9]; (ii) at frequencies for which the EFSs are centred in the $\Gamma$ point and become rounded with their radii diminishing with increasing frequency [7]. The first case is common in the first photonic band just below the band gap whereas the second case takes place at higher photonic bands. In the second case, the PhC can mimic a NIM in terms of refraction if the interface termination of the $\mathrm{PhC}$ is properly chosen [10-13] although in terms of EM propagation along the medium the $\mathrm{PhC}$ behaves well different from the ideal NIM proposed by Veselago [14], being the evolution of the EM wave phase fronts always positive even in a region in which negative refraction takes place [15].

Subwavelength imaging in two dimensional (2D) PhCs has been studied at frequencies accomplishing condition (i) [16-19] as well as condition (ii) [20-27]. In the first case, the $\mathrm{PhC}$ does not behave as an isotropic NIM as proposed by Veselago and the imaging occurs only in the near-field [16, 17] and placing the source very close to the PhC slab unless the unit cell of the $\mathrm{PhC}$ is properly engineered so that the $\mathrm{PhC}$ behaves as an isotropic medium [18, 19]. In the case given by condition (ii) the $\mathrm{PhC}$ can behave as an isotropic medium if the orientation and the surface termination are properly chosen [10-13] and the imaging follows the rules of geometric optics as in the case of the NIM slab studied by 
Veselago. Thus, by use of PhCs designed to provide negative refraction (isotropic or anisotropic) it becomes feasible to correct the phase of PWs originated in a point source and create an image at the other side of the PhC slab. This is schematically shown in Fig. (1) (the direction of the PW is depicted as a red arrow). EFS are depicted both in vacuum and in the PhC. The EFSs are rounded (isotropic behaviour) and their radius (equal to $2 \pi f / c, f$ being the absolute frequency and $c$ the light speed in vacuum) is the same for both media, which means that the effective index in the $\mathrm{PhC}$ is -1 . In the PhC, the EFS shrinks when increasing the frequency, resulting in negative refraction of PWs [8]. In addition, the EFSs in the reciprocal space are replicated along the transverse wave vectors $\left(k_{\mathrm{x}}\right)$ with period $2 \pi / p, p$ being the interface period [10]. However, the condition of conservation of the tangential component of the incident wave vectors only produces a refracted ray for the fundamental PhC EFS centred on $k_{\mathrm{x}}=0, k_{\mathrm{z}}=0$, which is inside the first Brillouin zone ($\left.\pi / p<k_{\mathrm{x}}<\pi / p\right)$.

This is the mechanism that explains how PWs are negatively refracted when crossing from vacuum to the $\mathrm{PhC}$ and vice versa. However, in order to obtain subwavelength imaging it is mandatory to restore, at least partly, the amplitude of the EWs emanating from the source, which contain the information related to the smallest details of the source. In a hypothetical NIM with $\varepsilon=\mu=-1$, EWs are amplified [2]. In addition, the excitation of surface plasmon polaritons (SPPs) can aid the restoring of the amplitude of the EWs [2]. In the case of a PhC, although it behaves as a NIM with negative effective $\varepsilon$ and $\mu$ for PWs [28], for EWs the PhC behaves as a conventional medium (positive $\varepsilon$ and $\mu$ ) in contrast with the case of the hypothetical NIM [25]. Thus, EWs are not amplified but attenuated inside the $\mathrm{PhC}$ material. However, there can be other mechanisms that contribute to reduce the decay of the EWs so that subwavelength resolution is achieved. These mechanisms have something in common: all of them rely on a coupling of the EWs with some bound state of the $\mathrm{PhC}$ at the frequency under consideration. The restoration of EWs by a negative-index PhC flat slab can be due to: (a) coupling of the EWs to surface states of the PhC (similar to the SPPs in nanostructured metals) [16]; (b) coupling of EWs to slab-guided bound states (which depend on the slab thickness) [17] and (c) coupling of EWs to bulk-guided states in higher-order Brillouin zones [17]. It has to be noticed that coupling of input EWs to slab-guided bound states can only occur for PhCs slabs accomplishing condition (i). If the $\mathrm{PhC}$ slab is engineered to have an effective index of -1 (usually done for the second photonic band) there are not slab-guided bound states within the first Brillouin zone that can be excited by external EWs. Thus, in the case studied in this work, where condition (ii) is assumed, EWs can couple to $\mathrm{PhC}$ modes throughout mechanisms (a) and (c), which is schematically shown in Fig. (1).

In a conventional imaging system the resolution is limited by the wavelength of the light. The resolution of a negative-index $\mathrm{PhC}$ slab is limited by the period of the $\mathrm{PhC}$ and the thickness of the slab (in principle, the total decay of EWs will be lower for thinner slabs, despite to the fact that they can be partly amplified by coupling to slab states). In addition, it has to be considered that although the $\mathrm{PhC}$ slab is engineered to have a refractive index of -1 , its impedance will be different to that of vacuum, so there will be reflections at the vacuum-PhC interfaces [28]. This is also in contrast with the hypothetical NIM lens studied by Veselago and Pendry, which is impedance matched with vacuum. Thus, multiple reflections at the PhC slab sides have to be taken into account since they can have influence on the quality of the image, which is mainly characterised by its spatial width and its amplitude. It has to be mentioned that subwavelength imaging in the microwave regime has been experimentally demonstrated for both 2D [29] and three-dimensional PhCs [30]. In this work we carry out a thorough analysis of the imaging properties of a $2 \mathrm{D} \mathrm{PhC}$ properly engineered to display an effective refractive index $n=-1$ at a certain frequency. The effects of the slab thickness and termination cut on the quality of the image are studied by use of a 2D finitedifference time-domain (FDTD) method [31]. Our results show that the image sharpness is better for thinner slabs and for certain PhC terminations for which surface states similar to SPPs are excited by the EWs. The properties of these surface states are analyzed by means of the Plane Wave Expansion (PWE) method [31], commonly employed to calculate band structures of PhCs.

\section{DESCRIPTION OF THE IMAGING SYSTEM UNDER STUDY}

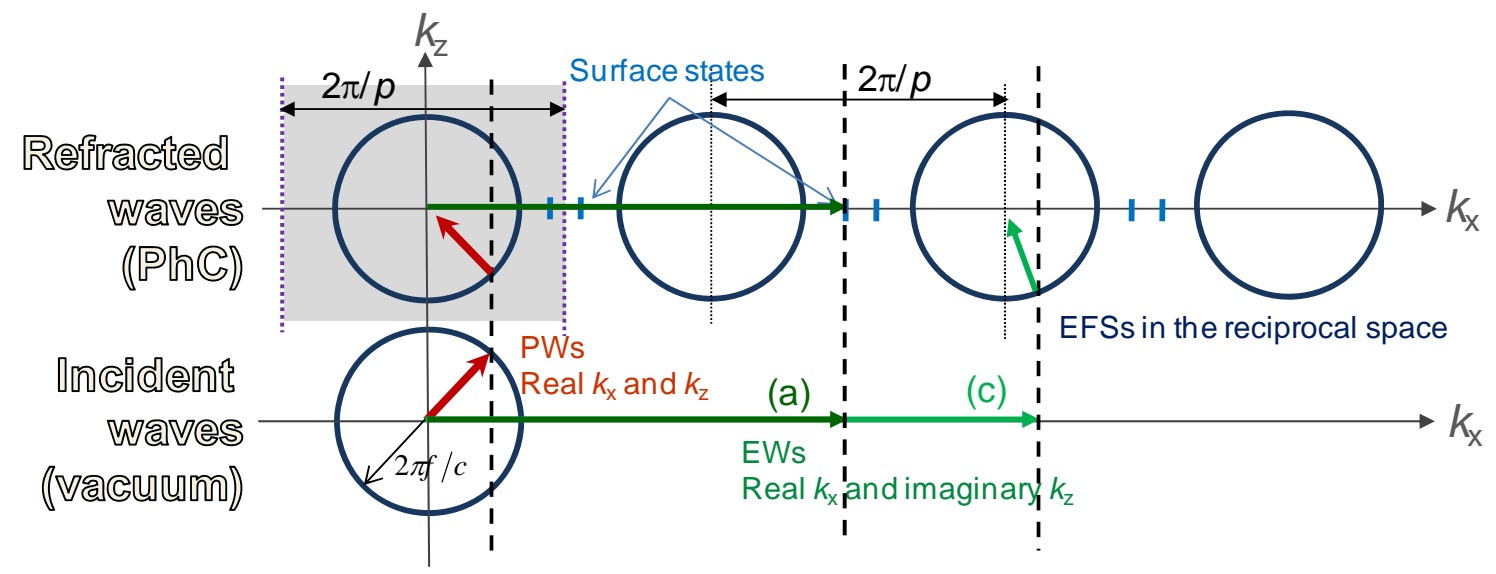

Fig. (1). Negative refraction for PWs and EWs passing from vacuum to a PhC. EWs in vacuum can be transformed into PWs in the PhC if coupled to a EFSs out of the first Brillouin zone (light gray shaded region). Vertical dashed lines stand for the conservation of the transverse wave vector $\left(k_{\mathrm{x}}\right)$ at the interface. 
The 2D PhC studied here consist of a hexagonal lattice of circular holes (radius $r=0.4 a, a$ being the lattice constant) embedded in a material with $\varepsilon=12.96$ [27]. We only consider TM modes (electric field parallel to the cylinders). The band structure in the first Brillouin zone of the $2 \mathrm{D} \mathrm{PhC}$ can be obtained by using the PWE method. When approaching the $\Gamma$ symmetry point the EFSs of the second photonic band become circular and their radius decrease with frequency, as can be seen in Fig. (2). At these frequencies the 2D PhC behaves as an effective medium with negative $n$ [7]. We obtained $n=-1$ at a frequency of 0.305 (in normalized units of $f a / c)$.

In principle, if both the orientation and the interface termination of the $\mathrm{PhC}$ are properly chosen [10-13], a 2D PhC slab of thickness $L$ should produce an image of a point source with frequency 0.305 allocated at a distance $d$ of the slab at a distance $L-d$ just at the other side of the slab [1]. Subwavelength imaging can be achieved if some of the EWs produced by the source are restored by some of the mechanisms described above for a PhC with $n=-1$ : the excitation of SPP-like surface states or the coupling of EWs to bulkguided states in higher-order Brillouin zones. In this work we focus on the first mechanism since the second always occurs. All the EWs whose wave vector lies in a higher-order Brillouin zone couple to a bulk-guided state increasing the resolution as more $\mathrm{EW}$ are restored.

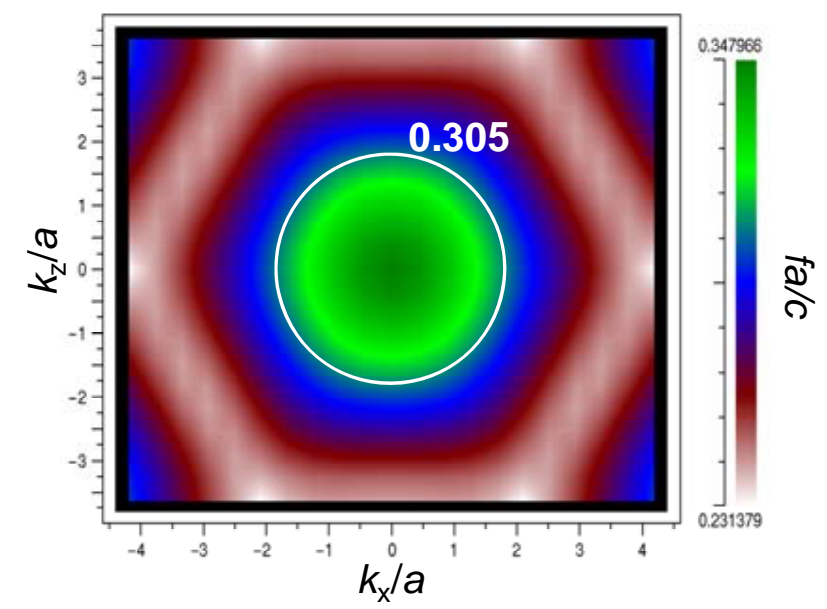

Fig. (2). Colour map of the EFSs of the $2^{\text {nd }}$ photonic band of the $2 D$ $\mathrm{PhC}$ under study (TM polarization) obtained by using the PWE method.

Although the $\mathrm{PhC}$ is designed to have an effective index of -1 , the slab is not necessarily matched in impedance with vacuum. In general, there will be reflections at the input and output interfaces of the $\mathrm{PhC}$ slab that produce several undesired effects as the reduction of the intensity of the image and the appearance of multiple low-intensity foci [32]. In addition, the image quality is not improved by the effect of the impedance mismatching as no loss dielectric material is considered in the system [33].

The imaging set-up employed in our numerical study is depicted in Fig. (3). We consider that the $2 \mathrm{D} \mathrm{PhC}$ slab is oriented so that the vacuum-PhC interfaces are parallel to the $\Gamma K$ direction. Thus, the interface has a period $p=a$ and symmetry matching of waves impinging from air normal to the interface is ensured for the second band [34]. We consider that the $\mathrm{PhC}$ slab has a thickness $L_{m}=12 \sqrt{ } 3 a-m \delta z$, where $\delta z=\sqrt{ } 3 a / 10$. The parameter $m$ is varied from 0 to 105 to change the slab thickness as well as the distance from the point source to the input interface. For higher values of $m$ the slab was too thin and a well-defined image was not formed. The maximum thickness of the $\mathrm{PhC}$ slab is $L_{m}=12 \sqrt{ } 3 a$, which is about 6.3 times the vacuum wavelength at the frequency under consideration. This means that for low values of $m$ we can consider that the imaging occurs at the far-field whereas for large values of $m$, the image will be produced in the near-field. The slab is allocated so that there is a hole centred at the origin, as shown in Fig. (3). In addition, the slab is kept symmetric with regard to the $z$ axis in order to ensure that terminations are identical at both sides of the slab regardless of $m$. The interface terminations have a periodicity of $\sqrt{ } 3 a$. In other words, input EM waves see the same interface termination for $L_{m 1}$ than for $L_{m 2}$ if $m_{2}=m_{1}+10 l, l$ being an integer, although if $l$ is odd there is an $a / 2$ displacement of the surface termination along the $x$ direction due to the hexagonal symmetry of the lattice. The length of the slab in the $x$ direction is $100 a$, which is large enough to assume an infinite aperture of the slab.

The transversal and longitudinal sizes of the output image are calculated by monitoring the electric field along both directions, as depicted in Fig. (3). Since the 2D PhC theoretically behaves as an effective index medium with $n=-1$ the position of the image should be at $x=0$ and $z=L_{m}$, following simple rules of geometric optics if only PWs are taken into account [1]. This case is in contrast with imaging at frequencies within the first photonic band, when the $\mathrm{PhC}$ does not behave as an effective medium and the image is in the nearfield region of the output interface (i.e., this imaging does not follow the rules of geometric optics since it is mainly due to amplification of EWs). However, it is worthwhile mentioning that the actual slab thickness that PWs experience when travelling across the slab can be different from $L_{m}$ due to the lateral Goos-Hänchen shift [10]. This will result in the formation of a well-defined image at a value of $z$ different from $L_{m}$, as will be shown below. In addition, the partial restoring of EWs will be also affect the position of the image along the $z$ axis, mainly when the slab is working in the nearfield regime [16].

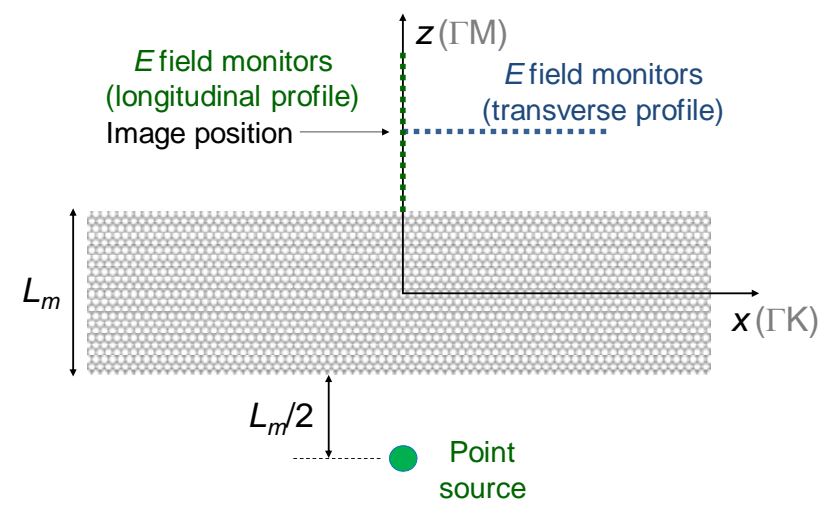

Fig. (3). Scheme of the imaging system studied in the FDTD simulations. 


\section{ACHIEVING A STEADY STATE RESPONSE IN FI- NITE-DIFFERENCE TIME-DOMAIN SIMULATIONS}

In the study of the imaging properties of the proposed 2D $\mathrm{PhC}$ slab we used a 2D FDTD method. In order to analyze the properties of the image produced by the imaging system depicted in Fig. (3) we need to obtain the EM response of the system in the steady state. In our simulations, the EM source is switched on at a time $t=0$ (we express time in normalized units of $c T / a, T$ being the real time from the beginning of the simulation). PWs need a certain amount of time to reach the input side of the slab, be refracted, propagate inside the slab, exit the lens and form a well defined image (maximum of field intensity at a positive value of $z$ ) of the source. This time is different depending on the angle for which a ray impinges the slab: rays with larger incident angles will need longer time to reach the point in which the image is formed. In addition, EWs could be restored inside the slab in order to achieve subwavelength focusing if bound slab modes exist at the frequency under study, and they will also need a finite amount of time to be present at the point of imaging. Thus, we have to know after which amount of simulation time a quasi-steady state has been reached and the image is well formed at the output of the slab. To this end we have carried out a very long FDTD simulation for the maximum thickness of the slab $L_{0}$ in order to obtain a value for the amount of time needed to reach a quasi-steady state. For thinner slabs, this quasi-steady state regime should be reached at a smaller value of time, so the time obtained for the slab with $L_{0}$ is also useful for slabs with $m>0$.

Fig. (4) shows the square electric field monitored at the slab output in the longitudinal direction as a function of the simulation time and the $z$ coordinate. Note that due to infinite reflections at the slab interfaces a true steady state would need an infinite simulation time to be reached. However, it can be observed that after a certain simulation time the position and width of the image remain almost unchanged to values $z / a=21$ and full-width at half-maximum, FWHM $=0.520422 \lambda$. (in the transverse direction). Roughly, we can consider that this time is $t=400$. Since the slab thickness is $L_{0}=20.78 a$, the image position for a hypothetical NIM lens should be $z=L_{0}$. The slight difference between the image position obtained with FDTD and the geometric optics prediction can be considered as a result of the difficulty in defining the interface between the $\mathrm{PhC}$ and the vacuum since the $\mathrm{PhC}$ is an inhomogeneous medium, the existence of the Goos-Hänchen effect at interfaces and the fact that the PhC EFS is not perfectly circular [27]. However, the agreement between the FDTD result and the ray optics analysis of a NIM slab is quite good.

It can be also observed that a secondary image is formed at large simulation times around $z / a=15$. This image gets well defined for a simulation time above $t=900$. However, since our aim is the analysis of the properties of the main image, we will consider that the image gets well defined for a time $t=400$ so this is the stop time that was used when calculating the imaging properties of the $\mathrm{PhC}$ slab with different values of $m$.

\section{FDTD RESULTS}

The longitudinal as well as the transversal image widths were estimated as a function of the slab thickness $L_{m}$. It was
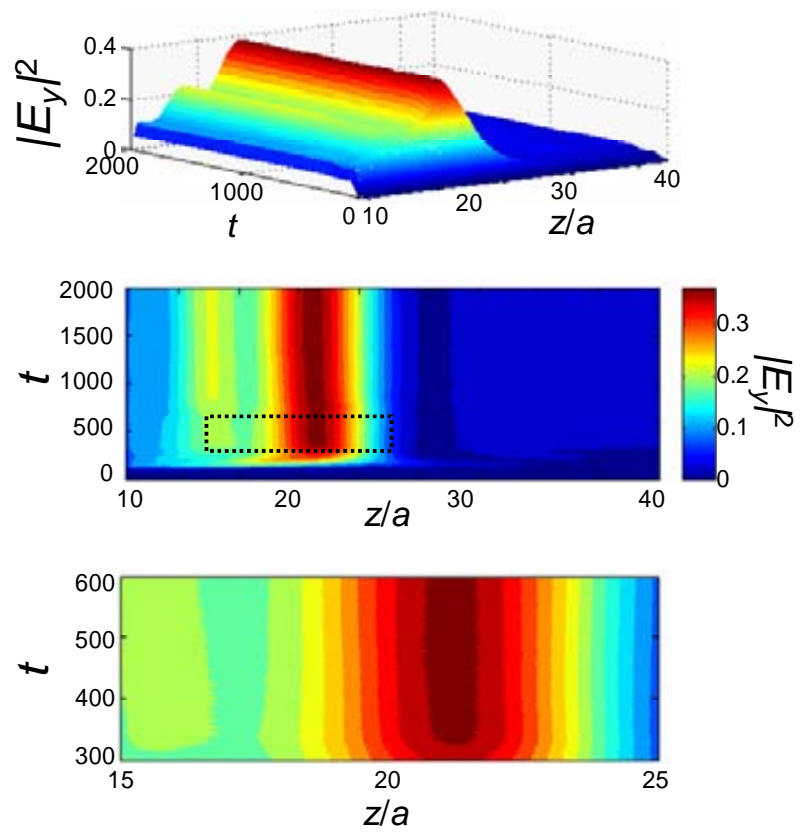

Fig. (4). Square electric field monitored at the slab output in the longitudinal direction as a function of the simulation time and the $z$ coordinate (upper and middle panel). The dotted region in the middle panel is enlarged for clarity (lower panel).

found that the position of the image was a bit different from the value $z=L_{m}$ expected from a simple geometric optics analysis. Fig. (5) depicts the image position normalized to $L_{m}$ (expected position) as a function of $m$. The image position displays an oscillatory behaviour around $z=L_{m}$ depending on $m$. When the slab is thin (high values of $m$ ) the ripples of the oscillations become larger. This can be explained by considering the existence of a shift of the refracted rays due to the Goos-Hänchen effect, which will strongly depend on the interface truncation (value of $m$ ) and can be quite high for a negative-index $\mathrm{PhC}$ [10]. A periodic-like behaviour in the response, which also occurs for other features of the image, is also observed. This is because the interface cut is the same for $m_{1}$ and $m_{2}=m_{1}+10 l, l$ being an integer. Fig. (6) shows the normalized peak intensity (a.u.) at the center of the image as a function of $m$. A periodic-like behaviour is again observed with intensity maxima close to unity at values of $m=7+10 l$ and minima at $m=4+10 l$. As we will show below, there is a direct relationship between the intensity maxima and minima and the excitation of surface states (SSs) at the slab interfaces. We find intensities higher than 1 for certain terminations. This can be explained by considering the restoration of EWs by coupling to SSs as explained below. The FWHM of the image in the transverse direction as a function of $m$ is shown in Fig. (7). The monitored FWHM is around $\lambda / 2$. However, subwavelength focusing $(\mathrm{FWHM}<\lambda / 2)$ is achieved at some slab cuts both in the far-field $\left(L_{m}>\lambda\right)$ and in the near-field regimes. The best resolution is found around $m=6+10 l$ and $m=7+10 l$, specifically at $m=76.4$

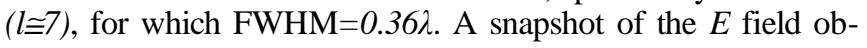
tained by FDTD for this case is depicted in Fig. (8).

\section{INFLUENCE OF SURFACE STATES ON SUBWAVE- LENGTH IMAGING}

To study the influence of SSs on the image resolution we used the PWE method. Fig. (9) shows the dispersion relation 


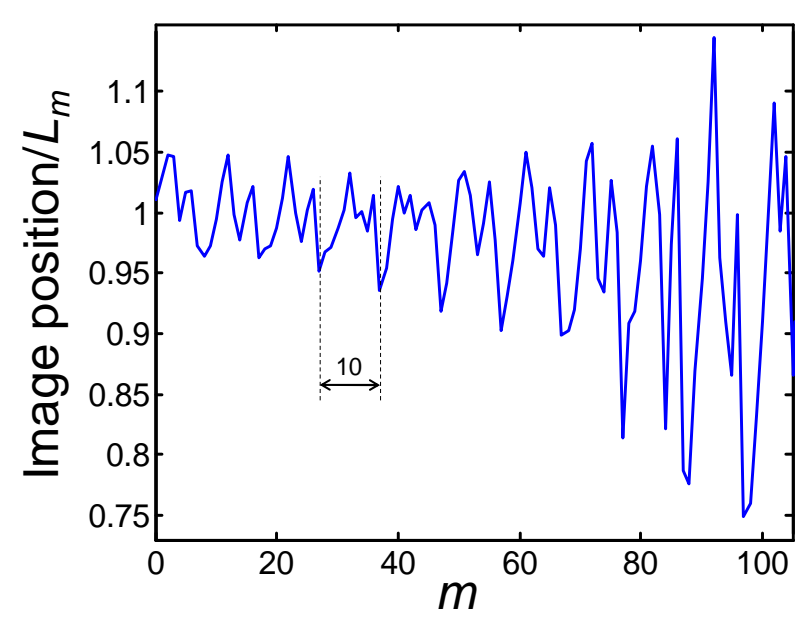

Fig. (5). Image position in the longitudinal direction.

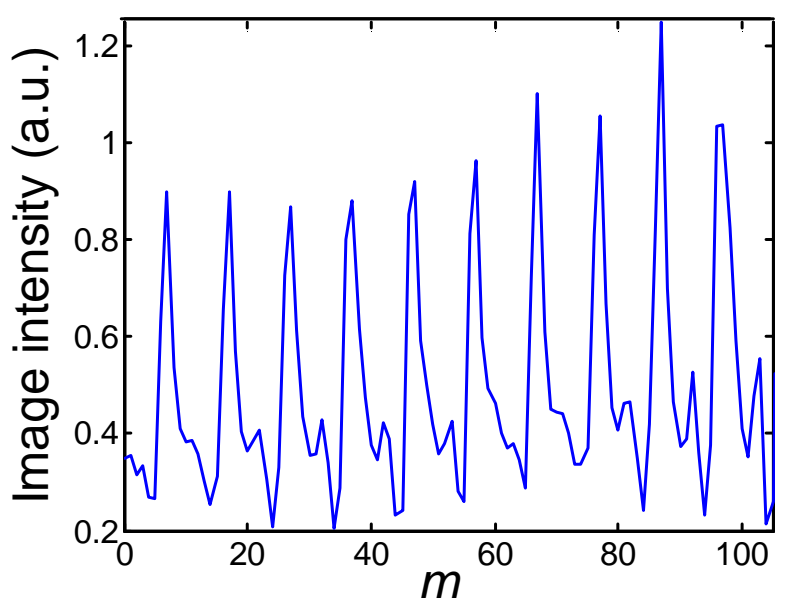

Fig. (6). Image intensity in its center position.

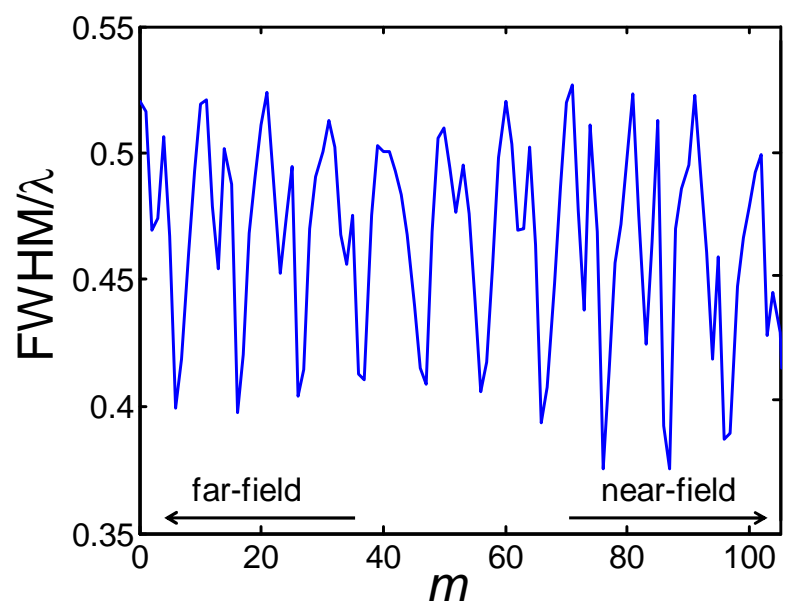

Fig. (7). Transverse FWHM as a function of $m$.

of the $\mathrm{PhC}$ for transverse wave vectors ( $x$ direction) and different terminations given by $m$. For the sake of clarity, only modes for integer values of $m$ are shown, but a continuum of SSs exists if non-integer values of $m$ are also considered. This behaviour can be easily drawn from the figure. For certain values of $m$, discrete modes appear at frequencies below

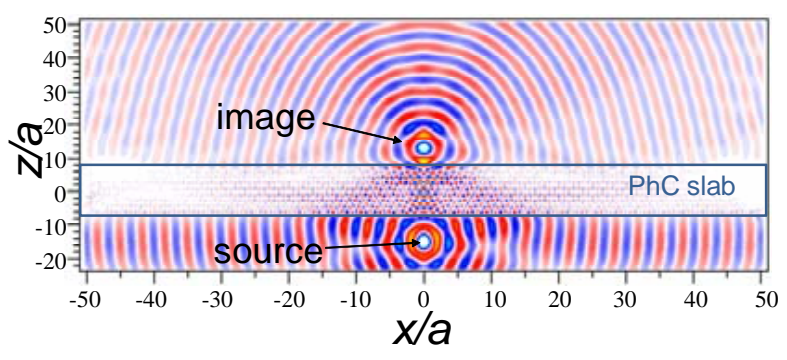

Fig. (8). Snapshot of the electric field obtained by FDTD for $m=76.4$ (FWHM=0.36 $\lambda$ ).

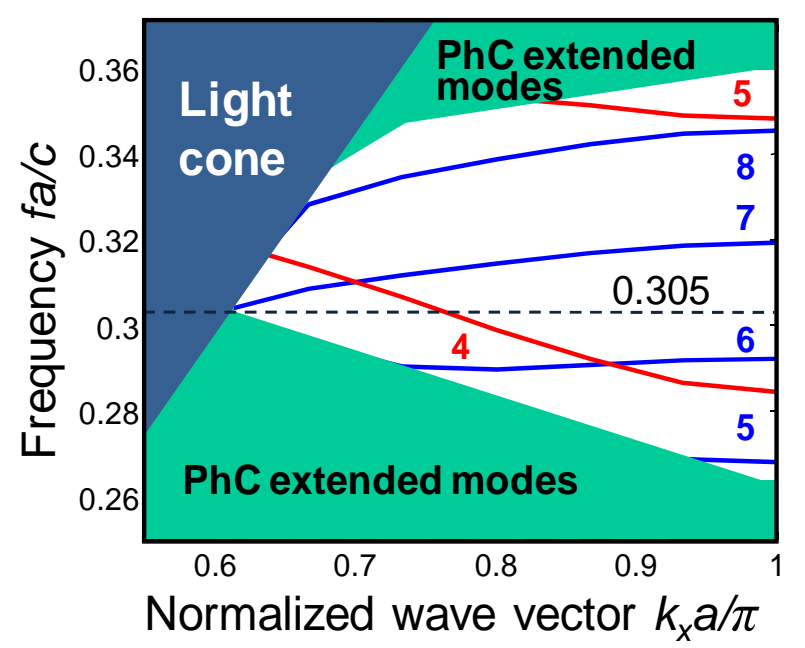

Fig. (9). Dispersion relation of the $2 \mathrm{D} \mathrm{PhC}$ along the $x$ axis for different values of $m$. SSs with even (blue) and odd (red) symmetries are appreciated.

the light cone for which no $\mathrm{PhC}$ extended modes exists. These modes are SSs bounded to the PhC surface. If a SS is close to the frequency under study, EWs can couple to this surface mode and can be partly restored at the image plane [16]. Depending on the lens thickness $L_{m}$, two kind of symmetry profiles of SSs are observed at frequencies close to 0.305 due to different interface termination of the PhC when varying $m$ : for $m=4$, the SS has an odd symmetry with respect the $z$ axis, as observed in the electric field pattern of Fig. (10). Owing to this reason, subwavelength imaging is not achieved when the interface is cut to support a SS with odd symmetry. For values of $m$ between 6 and 7 we can find SSs with even symmetry (Fig. 10) around the frequency 0.305 , which explains the subwavelength resolution for this termination even in the far-field as well as the large intensity detected at the image. This can be explained by considering that the source has even symmetry, which applies to both PWs and EWs. Thus, EWs can only couple (and thus, be partly restored at the image plane) to even-symmetry SSs. We can conclude that the symmetry of the SS plays a fundamental role in the formation of subwavelength images, which can be achieved even in the far-field by properly selecting the slab cut.

Notice that intensity minima (maxima) occur for terminations supporting odd (even) symmetry SSs. A maximum intensity in the far-field is mainly originated from low reflectivity of propagating waves for the corresponding interface 


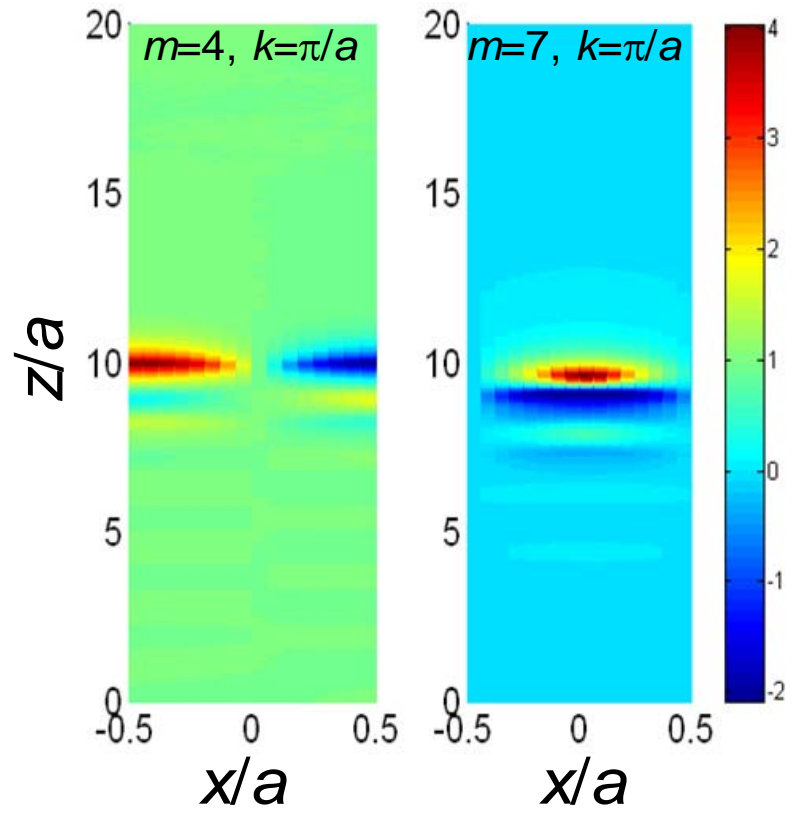

Fig. (10). E-field $\left(E_{y}\right)$ patterns of the odd (left) and even (right) symmetry PhC SSs.

cut. Thus, we can state that there is necessarily some relationship between image intensity and the SS supported by a certain PhC cut. We studied the reflectivity of the PhC interface for a plane wave of normalized frequency 0.305 impinging parallel to the $z$ axis by means of FDTD simulations. Results are shown in Fig. (11) for $m$ varying from 0 to 10 . It can be seen that the $m$ values which result in a higher (lower) reflectivity closely correspond to those with lower (higher) values of the imaging intensity and higher (smaller) values of the transversal FWHM. Thus, it seems that there is a direct relationship between low (high) reflectivity cuts with the existence of even (odd) symmetry SSs. In other words, an interface that supports a SS has effects on both EWs, which can couple or not to the SS depending on its symmetry, and PWs, which find a higher or lower impedance mismatching depending on the symmetry of the SS that can exist in the interface.

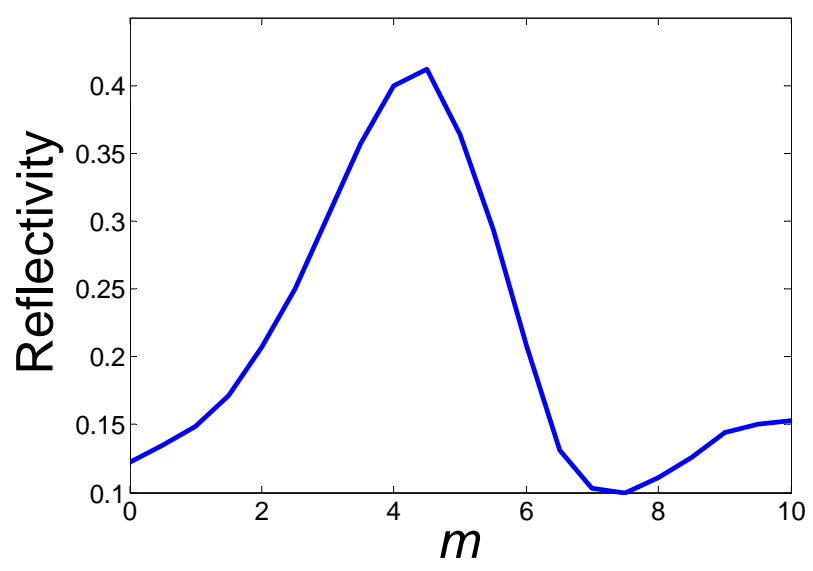

Fig. (11). Reflectivity of a PhC slab surface for different terminations ( $m$ values between 0 and 10).

\section{CONCLUSIONS}

We have shown by a numerical analysis that SSs for certain surface terminations of a $2 \mathrm{D} \mathrm{PhC}$ slab with an effective index equal to -1 can contribute to the partial restoration of EWs so subwavelength imaging can be achieved for both near and far-field. The position of the image is also strongly dependent of the $\mathrm{PhC}$ cut. In addition, it is shown that cuts with high (low) reflectivity are related to the existence of odd (even) symmetry SSs. Further analysis between the relationship between the SSs and the properties of the image formed at the output of the slab will be carried out.

\section{ACKNOWLEDGEMENTS}

Financial support by the Spanish MEC and EU-FEDER under contract TEC2005-06923-C03-03 and Generalitat Valenciana (grant ACOMP07/238) is acknowledged. R.O. acknowledges UPV for funding his grant.

\section{REFERENCES}

[1] Veselago VG. The electrodynamics of substances with simultaneously negative values of $\varepsilon$ and $\mu$. Sov Phys Usp 1968; 10: 594-514.

Pendry JB. Negative refraction makes a perfect lens. Phys Rev Lett 2000; 85: 3966-9.

[3] Garcia N, Nieto-Vesperinas M. Left-handed materials do not make a perfect lens. Phys Rev Lett 2002; 88: 207403. Pendry JB: Comment on Left-handed materials do not make a perfect lens. Phys Rev Lett 2003; 91: 099701.

[4] Pendry JB, Holden AJ, Robbins DJ, Stewart WJ. Magnetism from conductors and enhanced nonlinear phenomena. IEEE Trans Microw Tech 1999; 47: 2075-84.

[5] Pendry JB, Holden AJ, Stewart WJ, Youngs I. Extremely low frequency plasmons in metallic mesostructures. Phys Rev Lett 1996; 76: 4773-6.

[6] Shelby RA, Smith DR, Schultz S. Experimental verification of a negative index of refraction. Science 2001; 292: 77-9.

[7] Notomi M. Theory of light propagation in strongly modulated photonic crystals: refractionlike behaviour in the vicinity of the photonic band gap. Phys Rev B 2000; 62: 10696-705.

[8] Foteinopoulou S, Soukoulis CM. Negative refraction and lefthanded behaviour in two-dimensional photonic crystals. Phys Rev B 2003; 67: 235107 .

[9] Luo C, Johnson SG, Joannopoulos JD, Pendry JB. All-angle negative refraction whitout negative effective index. Phys Rev B 2002; 65: 201104.

[10] Martínez A, Martí J. Negative refraction in two-dimensional photonic crystals: role of lattice orientation and interface termination. Phys Rev B 2005; 71: 235115.

[11] Istrate E, Green AA, Sargent EH. Behavior of light at photonic crystal interfaces. Phys Rev B 2005; 71: 195122.

[12] Jiang W, Chen RT, Lu X. Theory of light refraction at the surface of a photonic crystal. Phys Rev B 2005; 71: 245115.

[13] Zhong Y, Wu L, Su H, Wong KM, Wang H. Fabrication of photonic crystals with tunable surface orientation by holographic lithography. Opt Express 2006; 14: 6837.

[14] Martínez A, Míguez H, Sánchez-Dehesa, J, Martí J. Analysis of wave propagation in a two-dimensional photonic crystal with negative index of refraction: plane wave decomposition of the Bloch modes. Opt Express 2005; 13: 4160-74.

[15] Martínez AJ, Martí J. Positive phase evolution of waves propagating along a photonic crystal with negative index of refraction," Opt Express 2006; 14, 9805-14.

[16] Luo C, Johnson SG, Joannopoulos JD, Pendry JB. Subwavelength imaging in photonic crystals. Phys Rev B 2003; 68: 045115.

[17] He S, Ruan Z, Chen L, Shen J. Focusing properties of a photonic crystal slab with negative refraction. Phys Rev B 2004; 70: 115113. Zhang $X$. Subwavelength far-field resolution in a square twodimensional photonic crystal. Phys Rev E 2005; 71: 037601.

[19] Hu X, Chan CT. Photonic crystals with silver nanowires as a nearinfrared superlens. Appl Phys Lett 2004; 85: 1520-2. 
[20] Xiao S, Qiu M, He S. Influence of the surface termination to the point imaging by a photonic crystal slab with negative refraction. Appl Phys Lett 2004; 85: 4269-71.

[21] Wang X, Ren ZF, Kempa K. Unrestricted superlensing in a triangular two-dimensional photonic crystal. Opt Express 2004; 12: 291924.

[22] Wang X, Kempa K. Negative refraction and subwavelength lensing in a polaritonic crystal. Phys Rev B 2005; 71: 233101.

[23] Smolyaninov II, Davis CC, Elliott J, Wurtz GA, Zayats AV. Superresolution optical microscopy based on photonic crystal materials. Phys Rev B 2005; 72: 085442.

[24] Zhang X. Effect interference and disorder on the far-field image in a two-dimensional photonic-crystal-based flat lens. Phys Rev B 2005; 71: 165116

[25] Li CY, Holt JM, Efros AL. Far-field imaging by the Veselago lens made of a photonic crystal. J Opt Soc Am B 2006; 23: 490-7.

[26] Li CY, Holt JM, Efros AL. Imaging by the Veselago lens upon a two-dimensional photonic crystal with triangular lattice. J Opt Soc Am B 2006; 23: 963-8.

[27] Martínez A, Martí J. Analysis of wave focusing inside a negativeindex photonic-crystal slab. Opt Express 2005; 13 2858-68.
[28] Decoopman T, Tayeb G, Enoch S, Maystre D, Gralak B. Photonic crystal lens: from negative refraction and negative index to negative permittivity and permeability. Phys Rev Lett 2006; 97: 073905

[29] Cubukcu E, Aydin K, Ozbay E, Foteinopolou S, Soukoulis CM. Subwavelength resolution in a two-dimensional photonic-crystalbased superlens. Phys Rev Lett 2003; 91: 207401.

[30] Lu Z, Murakowski JA, Schuetz CA, Shi S, Schneider GJ, Prather DW. Three-dimensional subwavelength imaging by a photoniccrystal flat lens using negative refraction at microwave frequencies. Phys Rev Lett 2005; 95: 153901.

[31] Calculations of photonic bands have been done with BandSolve ${ }^{\mathrm{TM}}$ which implements the PWE method, and FDTD simulations with Fullwave $^{\mathrm{TM}}$, both by RSoft Design Group.

[32] Pokrovski AL, Efros AL. Lens based on the use of left-handed materials. Appl Opt 2003; 42: 5701-5.

[33] Lee K, Park H, Kim J, Kang G, Kim K. Improved image quality of Ag slab near-field superlens with intrinsic loss of absorption. Opt Express 2008; 16: 1711

[34] Martinez A, Míguez H, Griol A, Martí J. Experimental and theoretical analysis of the self-focusing of light by a photonic crystal lens. Phys Rev B 2004; 69: 165119

This is an open access article licensed under the terms of the Creative Commons Attribution Non-Commercial License (http://creativecommons.org/licenses/by$\mathrm{nc} / 3.0 /$ ) which permits unrestricted, non-commercial use, distribution and reproduction in any medium, provided the work is properly cited. 